\title{
Photochemistry of metal coordination complexes: metal to ligand charge transfer excited states
}

\author{
Thamas J. Meyer \\ Department of Chemistry, The University of North Carolina, Chapel Hill, \\ N.C. 27514 , U.S.A. \\ Abstract - Systematic variations appear in the photophysical and \\ photochemical properties of MLCT excited states which can be accounted for \\ qualitatively or even quantitatively based on the properties of the \\ molecules and of the surrounding medium.
}

In 1959, Paris and Brandt first reported emission from the complex [Ru(bpy) $]^{2+}$ (bpy is 2,2'bipyridine) in solution (ref. 1). The excited state involved is sufficientiy long-lived ( $~ 800 \mathrm{~ns}$ in water at room temperature) that the possibility existed for using it as a chemical reagent and based on quenching studies using complexes of Co(III), Adamson and coworkers suggested that the excited state could act as a reducing agent (ref. 2). Their suggestion was soon verified by additional quenching and flash photolysis studies (ref. 3,4). It was also shown that the excited state could act as an oxidizing agent (ref. 5), that its redox potentials could be estimated experimentally by a kinetic quenching technique (ref. 6,7), and that it could undergo facile, bimolecular electron transfer with a variety of oxidants or reductants (ref. 8-10). For example, oxidative quenching by paraquat (PQ ${ }^{27}$,

\section{Scheme 1}

$\mathrm{Ru}(\mathrm{bpy})_{3}^{2+} \stackrel{\mathrm{h} \nu}{\longrightarrow} \mathrm{Ru}(\mathrm{bpy})_{3}^{2+*}$
$\mathrm{Ru}(\mathrm{bpy})_{3}^{2+*}+\mathrm{PQ}^{2+} \stackrel{\mathrm{k}_{\mathrm{q}}}{\longrightarrow} \mathrm{Ru}(\mathrm{bpy})_{3}^{3+}+\mathrm{PQ}^{+}$
$\mathrm{Ru}(\mathrm{bpy})_{3}^{3+}+\mathrm{PQ}^{+} \stackrel{\mathrm{b}_{\mathrm{b}}}{\longrightarrow} \mathrm{Ru}(\mathrm{bpy})_{3}^{2+}+\mathrm{PQ}^{2+}$

provides a basis for the conversion of visible light energy into a transiently stored redox pair. Similar electron transfer schemes had been established previously for organic excited states (ref. 11), but the high chemical stability of the Ru complex in more than one oxidation state and its high light absorptivity in the visible made it an attractive candidate for exploiting the energy conversion possibilities associated with Eq. 1-3.

The successful utilization of $\mathrm{Ru}(\mathrm{bpy})_{3}{ }^{2+}$ and related complexes in energy conversion schemes relies on establishing a basis for understanding the light absorptivity and photophysical and photochemical properties of the chromophores involved (ref. $9 a, 12$ ). The goal of this account is to describe those properties and to provide a basis for understanding them at the microscopic level.

\section{ELECTRONIC STRUCTURE. LIGHT ABSORPTIVITY}

$\left[\mathrm{Ru}(\mathrm{bpy})_{3}\right]^{2+}$ and $\left[\mathrm{Os}(\mathrm{phen})_{3}\right]^{2+}$ (phen is 1,10-phenanthroline) share with complexes like $\left.[\mathrm{Mo}(\mathrm{bpy})(\mathrm{C}))_{1}\right],\left[\operatorname{Re}(\mathrm{phen})(\mathrm{CO})_{3} \mathrm{Cl}\right]$, and $\left[\operatorname{Ir}(\mathrm{bpy})_{2} \mathrm{Cl}_{2}\right]^{+}$the ground state electronic configuration $(\mathrm{d} \pi)^{6}$ ( $t_{2}^{6}$ in 0 symetry) and vacant, low lying $\pi *$ (bpy or phen) orbitals on the polypyridyl ligands of appropriate symmetry to mix with the d $\mathrm{d}$ orbitals. Visible light absorption in these complexes is usually dominated by intense absorption bands arising from $\mathrm{d} \pi \rightarrow \pi^{*}$ metal to ligand charge transfer (MICT) transitions, e.g., Mo(bpy)(CO) $+\mathrm{h} \nu \rightarrow$ $\mathrm{MO}^{\mathrm{I}}(\mathrm{b} \overline{\mathrm{p}} \mathrm{y})(\mathrm{C}) \mathrm{A}^{\circ}$ For the low oxidation state complexes of $\mathrm{MO}(\mathrm{O})$ and $\operatorname{Re}(\mathrm{I})$ the carbonyl groups are necessary to mix with and stabilize the dm levels thus decreasing sensitivity towards oxidation and bringing the $d \pi-\pi *$ (bpy or phen) energy gap into the visible region of the spectrum. In the higher axidation state complexes of $\mathrm{Rh}$ (III) or Ir(III) the $\mathrm{d} \pi$ levels are so stabilized by the higher effective nuclear charge at the metal that the MLCT transitions occur at high energies and can overlap with $\pi \rightarrow \pi^{*}$ based transitions localized on the polypyridyl ligands. 
The most straightforward experimental evidence supporting the assignment of the visible absorption bands to MLCT transitions and of emission to the corresponding IMCT transitions comes from linear correlations between absorption or emission band energies and ground state reduction potentials for the appropriate metal and ligand-based couples (ref. 13,14) e.g. $\left(\mathrm{L}=\infty, \mathrm{CH}_{3} \mathrm{CN}, \mathrm{PR}_{3}, \ldots\right)$,

$$
\begin{aligned}
& \text { (bpy) Os }{ }^{I I I}(L)_{4}^{3+}+e \rightarrow(\text { bpy }) O s^{I I}(L)_{4}^{2+} \quad\left(E_{\alpha x}\right)^{2+} \\
& \left.(\text { bpy }) s^{I I}(L)_{4}^{2+}+e \rightarrow(b \bar{y} y) o s^{I I}(L)_{4}^{+} \quad \text { (E }{ }_{\text {red }}\right)
\end{aligned}
$$

Experimentally, linear correlations exist between $\triangle E_{j}\left(=E_{0 x}-E_{r e d}\right)$ and $E_{\text {abs }}$ or $E_{e m}$ as expected since, for example, $\triangle E_{1 / 2}$ is the energy quantfty for thedouter-sphese equivalent (bpy) Os ${ }^{\mathrm{III}}(\mathrm{L})_{4}^{3+}+(\text { bōy })_{\mathrm{Os}}^{\mathrm{II}}(\mathrm{L})_{4}^{+} \rightarrow 2$ (bpy)Os ${ }^{\mathrm{II}}(\mathrm{L})_{4}^{2+}$

of the intramolecular, IMCT process that corresponds to emission (ref. 14a). $\left(b_{\bar{p} y}\right)^{\mathrm{III}}(\mathrm{L})_{4}^{2+*} \rightarrow(\text { bpy })^{2+s^{I I}}(\mathrm{~L})_{4}^{2+}+\mathrm{h}$

A schematic orbital and state energy level diagram for $(\mathrm{d} \pi)^{6}$-polypyridyl complexes is shown in Fig. 1.

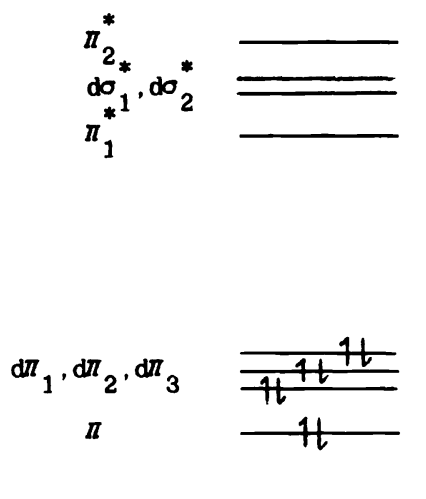

(Orbitals)

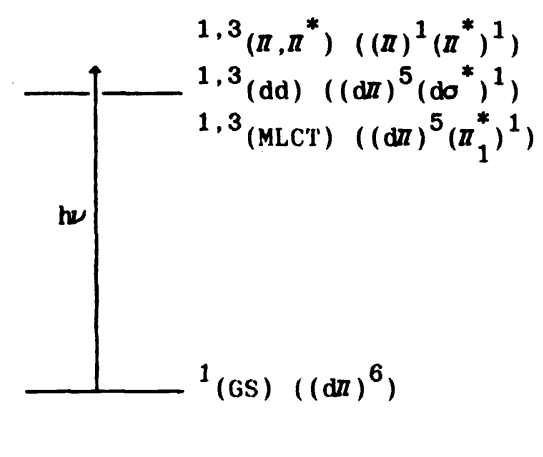

(States)

Fig. 1. A schematic orbital and state diagram for (d $\pi)^{6}$ MLCT excited states

A complex melange of excited states exists because of the presence of both filled $d \pi$ and $\pi$ (bpy or phen) levels and of unfilled $\pi^{*}$ and do* ( $e_{g}$ in 0 symmetry) levels. The relative disposition of the states and the state or states which oontrol photochemical and photophysical properties following excitation depend on the metal and its oxidation state, on the chromophoric ligand, on the additional, nonchromophoric ligands, and can even depend on the nature of the surrounding medium. However, in general:

1) Visible light absorption is usually dominated by transitions to MLCT excited states which are largely singlet in character ${ }^{1}\left[(d \pi)^{5}\left(\pi^{*}\right)^{1}\right]$ and emission occurs fram MLCT states which are largely triplet in character, $3_{[}\left[\left(d_{\pi}\right)^{5}\left(\pi^{*}\right)^{1}\right]$. The energies of the MLCT states vary systematically as the non-chromophoric ligands are varied. This is an expected result given the considerable difference in electronic configuration at the metal between the $(\mathrm{d} \pi)^{5}(\pi *)^{1}$ excited, and $(d \pi)^{6}$ ground states. For example, good backbonding ligands like $\infty$ stabilize $(\mathrm{d} \pi)^{6}-\mathrm{Ru}$ (II) by metal to ligand backbonding but not (d $)^{5}-\mathrm{Ru}$ (III) to any appreciable degree. consequently, incorporation of good backbondirig ligands leads to an increased energy gap between the excited and ground states. The change in electronic configuration between the $(d \pi)^{5}\left(\pi^{*}\right)^{1}$ excited and $(d \pi)^{6}$ ground states involves $d \pi$ orbitals which are largely nonbonding and only half population of anti-bonding $\pi^{*}$ (bpy or phen) orbitals. Consequently, relatively small molecular distortions in the excited states are expected to exist. However, given the significant change in radial distribution involved in the MLCT transition, absorption spectra and the energies of equilibrated MLCT excited states are expected to be medium dependent.

2) In the dd excited states $(d \pi)^{5}\left(d a^{*}\right)^{1}$, there is a significant increase in electronic repulsion along the metal ligand sigma bonding axes because of the promotion of a largely non-bonding electron to a sigma anti-bonding do-* orbital (ref. 15). Because of their LaPorte forbiddenness, absorption bands arising from $d \rightarrow d$ transitions are weak and not generally observed in the absorption spectra of $(\mathrm{d} \pi)^{6}$ polypyridy 1 complexes. In less spectroscopicaily cluttered camplexes of ruthenium, dd-bauls appear at relatively high 
energies, e.g., < $400 \mathrm{~mm}$ for $\left[\mathrm{Ru}\left(\mathrm{NH}_{3}\right)_{6}\right]^{2+}$ (ref. 16). However, because of the significant differences in structure along the metal-ligand bond axes between the $(d \pi)^{6}$ and $(d \pi)^{5}\left(d_{0-*}\right)^{1}$ configurations, when thermally equilibrated, dd excited states appear at much lower energies. If accessible they can play a significant role in the photochemical and photophysical properties of a complex since they are relatively short lived in solution and are often the source of photochemical instability arising from ligand loss. The energies of dd excited states above the ground state in analogous complexes increase approximately $\sim 308$ in passing from the first transition series to the second and $\sim 308$ again from the second to the third (ref, 17,18 ). It is for this reason that MLCT excited states based on Fe(II) are essentially unexploitable in energy conversion applications because dd states lie lowest, why the photophysical properties of ruthenium complexes are sometimes quite complex because the energies of MLCT and dd state are comparable, and is one of the endearing properties of complexes of $\mathrm{OS}$ (II) where dd states are often sufficiently high in energy that they play no role.

3) $\pi-\pi^{*}$ states-Absorption bands arising from polypyridine localized $\pi \rightarrow \pi$ transitions appear to vary somewhat in energy with the metal and its oxidation state but generally appear at $\sim 300 \mathrm{~mm}\left(\pi \rightarrow \pi^{*}{ }_{1}\right)$ and $\sim 240 \mathrm{~mm}\left(\pi \rightarrow \pi^{*}{ }_{2}\right)$. Because of the lack of dipole character for the $\pi \rightarrow \pi^{*}$ transition, $\pi-\pi^{*}$ excited states, are relatively insensitive to solvent variations and at low temperatures vibrational structure arising from aromatic ring based stretching modes can appear in their emission spectra (ref. 19). Polypyridine based $\pi-\pi$ * excited states are nearly always at higher energies than MLCT excited states except for cases like $\mathrm{Rh}$ (III) where the relatively high oxidation state stahjlizes the d $\pi$ levels below $\pi$ (bpy or phen) (ref. $12 a, 20,21$ ) or for complexes like [Ru(biq) $]^{27}$ (biq is bis-isoquinoline) where stabilization of the $d \pi$ orbitals by metal to ligand backbonding leads to $\pi-\pi^{\star}<M L C T$ (ref. 19).

A considerable complexity exists in the MLCT spectral region (ref. 22) arising from a number of factors: 1) In the MLCT excited state, $(d \pi)^{5}\left(\pi^{*}\right)^{1}$, the three $d \pi$ orbitals are mixed and further split by low symetry and spin orbit coupling. 2) At the polypyridyl ligands electronic interactions between ligands can cause a splitting amongst the $\pi *$ level. 3) Even in single polypyridyl-based chromophores, the polypyridyl ligand has a series of $\pi *$ levels which can provide the basis for multiple MLCT transitions. 4) There are both MLCT singlet and triplet states. Although the triplet transitions, ${ }^{l}\left[(d \pi)^{6}\right] \rightarrow{ }^{3}\left[(d \pi)^{5}(\pi *)^{1}\right]$, are forbidden in the absence of spin-orbin coupling, they become allowed when spin orbit coupling is included. From the theoretical point of view the effect of spin orbit coupling is to "mix" pure singlet and triplet states which imparts singlet character and allowedness to transitions involving triplets.

Typically, absorption bands in the $\mathrm{UV}$ and visible are observed arising from $d \pi \rightarrow \pi \star$ and $d \pi$ $\rightarrow \pi *$ transitions. The electronic spectra can be quite complex as illustrated by the low temperature single crystal spectra of $\mathrm{M}(\mathrm{bpy})_{3}^{2+}(\mathrm{M}=\mathrm{Fe}, \mathrm{Ru}, \mathrm{Os})$ obtained by Ferguson, et. al. (ref. 23). In the spectra many absorption features can be observed in the $d^{\pi} \rightarrow \pi *$ region. The various spectral features can be assigned to a series of MLCT transitions to both $1_{\text {MLCT }}$ and 3 MLCT states and to their vibronic components based on a parameterized molecular orbital model which includes the effects of spin orbit coupling (ref. 24,25 ). The appearance of transitions to "3MLCT" states at low energies is especially pronounced for os where the large spin orbit coupling constant $\left(\lambda \sim 3000 \mathrm{~cm}^{-1}\right)$ ensures significant singlet-triplet mixing. Analysis of the spectra using the molecular orbital model shows that the lowest energy MLCT states arise from a common set of $\mathrm{d} \pi$ and $\pi *$ orbitals, are largely triplet in character, and are mixed to different extents with low lying MLCT singlet states.

\section{Electronic structure. What happens following optical excitation? For $\mathrm{Ru}(\mathrm{bpy})_{3}^{2+}$ optical} excitation throughout the $\mathrm{d} \pi \rightarrow \pi^{*}$ spectral region results in the rapid, $<1$ ps (ref. 26), quantitative appearance of an emitting ${ }^{3} \mathrm{MLCT}$ state or states (ref. 27) whose emission is somewhat medium dependent but occurs around $600 \mathrm{rm}$. From absorption spectral measurements the energy gap between the lowest $l_{\text {MLCT }}$ and 3MCT states is only $\sim 500 \mathrm{~cm}^{-1}$. Since the "singlet" and "triplet" states are mixed by spin-orbit coupling, it is not surprising that excitation into the $1_{M L C T}$ absorption manifold leads rapidly to the emitting $3_{M L C T}$ state or states.

However, an interesting question remains in multiple chelates. Is the excited electron localized on a single ligand as in $\left[(\text { bpy }){ }_{2} \mathrm{Ru}^{\mathrm{III}}(\mathrm{b} \dot{p} \mathrm{y})\right]^{2+^{*}}$, possibly with rapid exchange occurring between ligands, or does the extited electron 'occupy a molecular orbital which is delocalized over all three ligands as in [RuIII (bpy - $1 / 3$ ) ${ }_{3}{ }^{2+*}$ ? In fluid solution, the excited electron in the emitting excited state(s) appears to be "localized" based on a number of lines of evidence: 1) Transient Resonance Raman spectroscopy where discrete, frequency shifted Raman lines are observed in the $v$ (bpy) ring stretching region (1000-1500 $\mathrm{cm}^{-1}$ ) for both reduced and unreduced bpy ligands (ref. 28,29). 2) Site selection spectroscopy based on emission polarization measurements (ref. 30). 3) Excited state correlations for related mono, bis, and tris-chelates like [Os (bpy) $]^{2+}$, [Os(bpy) (cis$\left.\left.\mathrm{Ph}_{2} \mathrm{PCH}=\mathrm{CHPPh}{ }_{2}\right)\right]^{2+}$, and $\left[\mathrm{Os}(\mathrm{bpy})\left(\text { cis- }-\mathrm{Ph}_{2} \mathrm{PCH}=\mathrm{CHPPh}_{2}\right)_{2}\right]^{2+}$ which have closely correlated excited 
state properties without differences arising which oould be attributed to delocaljzation effects (ref. 31). 4) In related ligand reduced complexes like [(bpy) $\left.{ }_{2}^{R u} I^{(b p j y)}\right]^{+}$generated electrochemically, there is clear spectral evidence that the added electron is localized on a single ligand and from epr line broadening that the rate of electron hopping between ligands is rapid (ref. 32,33 ).

The experimental evidence for localization in fluid solution is onvincing but the situation is less clear in the solid state or in low temperature glasses where the evidence remains somewhat equivocal (ref. 34,35 ). The question of localization versus delocalization is really a problem in mixed-valence chemistry where the issues involved have been discussed in some detail and applied mainly to ligand-bridged, mixed valence dimers (ref. $10 \mathrm{a}, 36,37$ ). The critical factors are the relative magnitudes of the electronic coupling between the electron donor (bojy) and acceptor (bpy) sites, $v$, and the vibrational trapping energy, $x$. vibrational trapping arises from the structural differences that exist between bị and bpy and the solvent dipole reorientations that must occur for electron hopping to ocur. From the theoretical point of view delocalization occurs when $v>x / 2$ (ref. 36). Fram spectroscopic measurements on $\left[\mathrm{Ru}(\mathrm{bpy})_{3}\right]^{2+}$ and related complexes it is clear that nonneglible contributions to_yibrational trapping exist from the solvent in polar organic solvents $\left(X \sim 600-800 \mathrm{~cm}^{-1}\right)$. Even in the solid state or in a frozen solution where solvent dipole trapping can not occur, contributions from intramolecular vibrational trapping exist and there is no guarantee that sufficient electronic coupling between bpy and bipy exists in the excited state for delocalization to occur.

Experimental evidence is available showing that in fluid solution the electron is initially localized furing the electronic transition. The ground states of complexes like [Ru(bpy) $]^{2+}$ or $\left[\mathrm{Os}(\text { bpy) }]^{2+}\right.$ have $\mathrm{D}_{3}$ symetry and no permanent dipole moment. If optical excitation produces a delocalized excited state, the $D_{3}$ symmetry of the ground state would be preserved and MLCT absorption band energies should be independent of the solvent. on the other hand, if excitation leads to an electron localized on a single ligand, a dipole is induced in the excited state and from dielectric continuum theory (ref. 38) the energies of MLCT transitions are predicted to vary with the optical dielectric constant of the solvent, $D_{\text {op }}\left(n^{2}\right)$, as (ref. 39$)$,

$$
\Delta E_{T}=\frac{\mu^{2}}{a^{3}}\left(\frac{1-D_{o p}}{2 D_{O P}+1}\right)
$$

In Eq. $8 \wedge E_{T}$ is the solvent induced energy shift in the band maximum, $\mu$ the dipole moment

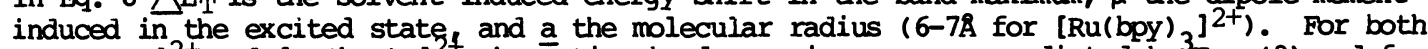
$\left[\mathrm{Ru}(\mathrm{bpy})_{3}\right]^{2+}$ and [Os(bpy) $]^{24}$ absorption band energies vary as predicted by $3_{\mathrm{Eq}}$. (8) and from the slopes of the lines it can be estimated that the dipole moments induced in the excited states are $\mu \cong 14 \pm 6$ D (ref. 39 ).

Low temperature measurements. Evidence for multiple MLCT excited states From the results of temperature dependent emission and lifetime studies, where the contributions of Crosby and $\infty$-workers are notable, (ref. 40,41 ) and more recent emission polarization experiments (ref. 34a) it is clear that following MLCT excitation, contributions to excited state properties exist from a series of states. In particular, below $77 \mathrm{~K}$ there is clear evidence for contributions to excited state decay and emission from three low-lying MLCT states based on changes in emission quantum yields, lifetimes and emission polarization as the temperature is increased above $4 \mathrm{~K}$.

Experimental evidence also exists for a fourth MLCT state at $\sim 600 \mathrm{~cm}^{-1}$ above the lowest state which is considerably shorter lived. For [Ru(bpy) $]_{3}^{2+}$ the existence of a fourth state is obscured in temperature dependent lifetime measurements in solution because of the intervention of a strongly temperature dependent transition to a low-lying dd state or states that become important above 200K. However, for [Os(bpy) $\left.{ }_{3}\right]^{2+}$ and related complexes of Os(II), temperature dependent lifetime studies provide direct ${ }^{3}$ evidence for the participation of a fourth state in excited state decay at higher temperatures $($ ref. 41,42$)$. Evidence for a fourth state for [Ru(bpy) $]_{3}$ 2f has also been obtained from temperature dependent emission polarization experiments (ref. 34a).

A parameterized molecular orbital model which includes spin orbit coupling and assumes localization of the excited electron has been applied to the MLCT excited states of $\left[\mathrm{Ru}(\mathrm{bpy})_{2}\right]^{2+}$ and [Os(bpy) $]^{2+}$ (ref. 44). The model correctly predicts the existence of four low-lying MLCT excited states. It also predicts that for Ru the lowest three MLCT states have less than 118 singlet character, 268 for Os, while the fourth state is expected to have a higher percent of singlet character.

Where the data are available, a closely related pattern of excited states exists for equivalent complexes of $\mathrm{Ru}$ and $\mathrm{Os}$ and state by state comparisons are revealing (ref. 45). 
For example, from the Einstein transition probability for spontaneous emission, the radiative rate constant, $k^{\prime}$, in terms of the integrated emittegd intensity at energy $\bar{v}, I(\bar{v})$ is given by Eq. 9. The tefm $\left\langle\bar{v}-3,-I=\left(\int I(\bar{v}) d \bar{v}\right) /\left(\int I(\bar{v}) \bar{v}^{-3} \mathrm{~d}^{\bar{\nu}}\right) \sim \mathrm{E}^{3}\right.$ is the average value of $v^{-3}$ for the emission band corresponding to the radiative transition (ref. 46 ) and $\langle d\rangle=$ $\left\langle\psi_{e}\left|e r_{i}\right| \psi_{g}\right\rangle$ is the electric dipole transition moment integral.

$k_{r}=\left(64 \pi^{4} n^{3} / 3 h\right)\left\langle\bar{v}^{-3}\right\rangle^{-1}\langle d\rangle^{2}$

In Eg. $9 \psi_{\mathrm{e}}$ and $\psi_{\mathrm{g}}$ are the excited and ground state electronic wave functions and $\mathrm{n}$ is the refractive index of the medium. The magnitude of the electric dipole transition integral depends on the amount of mixing of higher lying singlet excited states into the emitting triplet excited states, $\psi_{e}=\alpha\left(I_{e}\right)+\left(1-\alpha^{2}\right)^{\frac{1}{2}}\left(3 \psi_{e}\right)$ where from first order perturbation theory, the mixing oefficient $\alpha$ is given by,

$\alpha=\left\langle{ }^{1} \psi_{e}\left|\mathrm{H}_{\mathrm{SO}}\right|^{3} \psi_{\mathrm{e}}\right\rangle /\left({ }_{\mathrm{E}}^{1} \mathrm{e}^{\left.-{ }^{3} \mathrm{E}_{\mathrm{e}}\right)}\right.$

In Eq. $10 \mathrm{H}_{\mathrm{SO}}$ is the spin orbit coupling operator and ${ }^{1} \mathrm{E}_{e},{ }^{3} \mathrm{E}_{\mathrm{e}}$ the energies of the pure, excited singlet and triplet states. Including the mixing coefficient, the transition dipole mament integral is given by,

$\langle d\rangle=\alpha\left\langle\psi_{e}\left|e_{i}\right|^{1} \psi_{g}\right\rangle$

The square of the mixing coefficient is proportional to the spin orbit coupling constant $\left(\lambda_{\mathrm{Ru}} \sim 1100 \mathrm{~cm}^{-1}\right.$; $\left.\lambda_{\mathrm{ss}} \sim 3000 \mathrm{~cm}^{-1}\right)$ and it follows from Eq. 9 that in comparing eqivalent states of $\mathrm{Ru}$ and Os,

$\frac{\left[k_{r} /\left(E_{e m}\right)^{3}\right]_{R u}}{\left[k_{r} /\left(E_{e m}\right)^{3}\right]_{O s}} \sim \frac{\left.\lambda_{R u}<^{1} \psi_{e}|e r|^{1} \psi_{g}\right\rangle_{R u}^{2}}{\lambda_{O s}\left\langle{ }^{1} \psi_{e}|e r|^{1} \psi_{g}\right\rangle_{\text {Os }}^{2}}$

Where the data are availazble, a state by state comparison shows that the ratio $\left[\left(\mathrm{k}_{\mathrm{r}} /\left(\mathrm{E}_{\mathrm{em}}\right)^{3}\right]_{\mathrm{Ru}} /\left[\mathrm{k}_{\mathrm{r}} /\left(\mathrm{E}_{\mathrm{em}}\right)^{3}\right]_{\mathrm{Os}}\right.$ is $\sim 1 / 3$, the ratio of the spin-orbit coupling constants (ref. 45). This observation clearly suggests that in terms of pure electronic effects the single most important difference between $\mathrm{Ru}$ and $\mathrm{Os}$ is the magnitude of the spin orbit coupling constant which detemines the singlet content of the low-lying, emitting MICT states.

The results of temperature dependent experiments clearly show that the "emitting MLCT excited state" of $\mathrm{Ru}$ (bpy) $3_{3}^{+}$and related complexes is a composite of states. Although all four lowlying states share a common $(d \pi)^{5}\left(\pi^{*}\right)$ electronic configuration, they are mixed to different extents with low lying excited singlet states and their energies and decay characteristics ${ }_{1}$ are different. The energy spacings amongst the three lowest states are small $\left(<200 \mathrm{~cm}^{-1}\right)$ and at temperatures near RT, to a good approximation, photophysical properties can be treated as rising from a single state having the averaged properties of the three contributors. The energy gap to the fourth state is higher, it is shorter lived because of its greater singlet character, and its population and decay can influence the temperature dependent properties of MLCT chromophores near roam temperature in solution.

\section{MOLECULAR STRUCTURE}

In $(d \pi)^{5}(\pi *)^{1}$ MLCT excited states, 1-electron occupation of the antibonding $\pi *$ orbital is expected to lead to structural changes in the chramophoric ligand based on known crystal structures of reduced or partly reduced polypyridyl ligands (ref. 47).

Structural differences between the ground and excited states can be resolved into a linear combination of the normal modes for which there is a change in frequency between states or, more importantly, for which the change in electronic distribution leads to a difference between states in the equilibrium displacement. The pattern of contributing normal modes can be discerned from Resonance Raman spectra by observing which modes are resonantly enhanced by scattering from MLCT transitions since resonance enhancements can only be observed for those nomal modes which respond to the change in electronic configuration between the states. Symmetry plays an important role. For example, if there is a symmetrical change in structure between the ground and excited states only those nomal modes which belong to the totally symetric representation of the point group can be involved in the interconversion between structures. For MLCT excited states, molecular orbital calculations have been used to predict the structural change upon excitation and to evaluate qualitatively the contributing normal modes (ref. 48).

From Resonance Raman experiments on Ru(bpy) ${ }_{3}^{2+}$ and related complexes, a series of 7-8 bpybased ring stretching modes are enhanced in the range 1000-1600 $\mathrm{cm}^{-1}$, a ring torsional mode 
at $\sim 600 \mathrm{~cm}^{-1}$ and a series of low frequency modes some of which can be assigned to metal ligand stretching vibrations (ref. 28,29,31,49). Additional information can be obtained from low temperaure emission spectra where well-defined vibrational progressions are often observed for MLCT emitters. The energy dependence of the emission intensity is given by (ref. 50,51 ),

$I_{v, v^{*}}(\bar{v})=\left(64 \pi^{4} c / 3 \hbar\right) N_{v^{*}}\left(\bar{v}_{v, v^{*}}\right)^{3}\langle d\rangle^{2} \pi\left\langle\chi_{j, v} \mid j, v^{*}\right\rangle^{2}$

In Eq. 13 the emission intensity as the number of quanta emitted per energy interval, $I_{\mathrm{v}, \mathrm{v}^{*}(\bar{v})}$, is given as the product of the number of molecules in the emitting state, $\mathrm{N}_{\mathrm{v}}$; the energy of the transition, $\bar{v}_{\mathrm{v}, \mathrm{v}^{*}}$, the speed of light, the transition dipole moment, and the product of the vibrational overlap integrals (Franck condon factors) for the contributing nomal modes. The product is over all the contributing modes, $j$, including solvent and other medium modes. $v$ and $v^{*}$ are the vibrational quantum numbers in the ground and excited states. Assuming the validity of the harmonic oscillator approximation, the contribution to emission from $a v^{*}=0$ level in the excited state to a level $v$ in the ground state is given by the square of the vibrational overlap integral (ref. 5l),

$\left\langle x_{v} \mid x_{v^{*}=0}\right\rangle^{2}=\frac{\left(S^{v}\right)}{v !} \exp (-s)$

In Eq. 14, $S$ is the electron-vibrational coupling constant which is a measure of the difference in equilibrium displacement between the ground and excited states, $\triangle \mathrm{Q} \cdot$ It is related to $\triangle \mathrm{Q}_{e}$ as shown in Eq. 15 where $M$ is the reduced mass for the vibration and $\omega(=2 \pi \nu)$ is the angular frequency.

$s=\frac{1}{2}\left(\frac{M \omega}{\hbar}\right)\left(\Delta Q_{e}\right)^{2}$

By using appropriate expressions for the vibrational overlap integrals in Eq. 13, it is possible to derive expressions for the emission spectral profile in terms of the properties of the contributing vibrations. For $\left[R u(b p y)_{3}\right]_{2+}$ it is necessary to include contributions from a pedium frequency mode with $\mathrm{h} \nu_{M}=h \nu_{M} \sim 1300-1400 \mathrm{~cm}^{-1}$, a low frequency mode with $h \omega \sim 300-$ $400 \mathrm{~cm}^{-1}$ and also contributions from solvent modes (dipole orientations) but treated semiclassically (ref. $31,46,52,53$ ). By comparing calculated and observed spectra, the emission spectral fitting procedure can be used to obtain values for the parameters, $S_{M^{\prime}} \hbar \omega_{M}, S_{L}, \hbar \omega_{L}, E_{O O}$, and FWHM. FWHM is the full width at half maximum (FWHM) for an individual vibrational component. The solvent enters through FWH as a broadening of the individual vibrational component and is related to $\chi$, the classical solvent vibrational trapping energy for the electron transfer process colresponding to the excited to ground state transition by (ref. 36 )

$X_{0}=\frac{(\text { FWHM })^{2}}{16 \mathrm{kTln} 2)}=\frac{(\mathrm{FWHM})^{2}}{2310}\left(\right.$ in $\mathrm{om}^{-1}$ at $\mathrm{RT}$ )

Given the evidence from Resonance Raman spectra for the participation of a series of medium and low frequency modes, experimentally derived values for $S_{y}, \hbar \omega_{M}$ are averages of contributions from 7 or 8 ring stretching modes and $S_{L^{\prime}}{ }^{h} \omega_{L}$ of a series of low frequency
modes including metal ligand stretching modes.

Application of the emission spectral fitting procedure to a series of polypyridyl complexes of $\mathrm{Ru}$ (II) and $O$ (II) (ref. $31,46,52,53$ ) have revealed: 1) Best fit values for $\hbar \omega_{\text {in }}$ in the range 1250-1400 $\mathrm{cm}^{-1}$ consistent with an averaged value for the contributing ring Modes observed by Resonance Raman. 2) Values of $S_{M}$ that increase linearly with $E_{\infty}$, the $v^{*}=0, v=0$ energy gap between the ground and excited states. The linear increase can be reconciled, at least qualitatively, as an increase in the extent of charge transfer between ground and excited states as $\mathrm{E}_{\infty}$ increases.

The experimentally derived values of $S_{y}$ lead to a direct approach for calculating excited state structures (ref. 31). An average $\mathrm{C}-\mathrm{N}, \mathrm{C}-\mathrm{C}$ bond distance change in the excited state compared to the ground state, $\Delta \bar{r}$, can be calculated from $S_{M}$ by,

$$
\Delta \bar{r}=\left(\frac{2 S}{b} \frac{\hbar}{M \omega}\right)^{\frac{1}{2}}
$$

where $\mathrm{b}$ is the number of $\mathrm{C}-\mathrm{C}$ and $\mathrm{C}-\mathrm{N}$ bonds in the ligand, $\mathrm{b}=13$ for bpy, and $M$ is the reduced mass. Applying this analysis to $\left[O s(b p y)_{3}\right]^{2+*}$ gives $\Delta^{\frac{1}{r}}=0.026 \pm 0.0005 \AA$.

Estimates for changes in average bond dispacements between the ground and excited states can also be obtained based on Badger's rule using the difference in vibrational frequencies between the ground and excited states obtained by ground state Resonance Raman and excited state transient Resonance Raman measurements (ref. 28,29). The relationship between the bond distance change, $\triangle r=r^{*} r$, and the frequency differences is shown in Eq. 18 .

$$
\Delta r=r^{*}-r=\ln \left(\nu_{v i b} N_{v i b}^{*}\right) / 2.45
$$


Based on frequency differences for five $\nu$ (bpy) modes from 1268-1610 $\mathrm{cm}^{-1}$, values of $\triangle \mathrm{r}$ in the range $0.0106-0.0173 \AA$ have been calculated in good agreement with $\triangle \bar{r}$ from emission spectral fitting (ref. 31). From the vibrational analyses, the bpy ring is not considerably distorted in the excited state. As expected, comparisons within a series of related complexes show that $\Delta \bar{r}$ increases with the excited to ground state energy gap consistent with a greater degree of charge transfer. One complication that is yet to be clearly resolved is whether $S_{M}, \hbar \omega_{M}$, etc. are significantly different for the three lowlying ${ }^{3} \mathrm{MCT}$ states. At or near room temperature the results obtained by emission spectral fitting are probably those of an averaged contribution from all three states.

\section{EXCITED STATE DECAY}

In many cases optical excitation of MLCT chromophores leads to the population of a series of low-lying MLCT states whose radiative and nonradiative properties dominate excited state decay at least at low temperatures. At higher temperatures additional, temperature dependent processes often appear which play an important role in helping to dictate the photochemical and photophysical properties of the complexes. Before turning to a detailed discussion of radiative and nonradiative decay from MLCT states, it is of value first to consider the competitive processes which appear at higher temperatures.

dd states In 1976 Van Houten and Watts studied luminescent lifetimes and emission quantum yields for $\left[R u(b p y)_{3}\right]^{2+}$ in water from 0 to $100^{\circ} \mathrm{C}$ (ref. 54). They found that they could fit the temperature dependent lifetime data to an expression of the form shown in equation 19 where $k$ was interpreted to be the sum of radiative, $k_{r^{\prime}}$ and nonradiative, $k_{n r}$ ' decay rate constants for the lowlying, "averaged" MLCT state.

$$
\tau(T)^{-1}=k+k^{\circ} \exp -\left(\triangle E^{\prime} / R T\right)
$$

In the interpretation given by Van Houten and Watts $\triangle E^{\prime}$ was the energy gap from MLCT to a

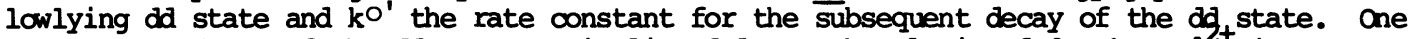
pathway for decay of the dd state(s) is ligand loss; photolysis of [Ru(bpy) $]_{3}{ }^{2}{ }$ in nonaqueous solvents in the presence of anions like $\mathrm{Cl}$ or NCS gives rise to photoinduced anation (ref. 55,56 ). Ligand loss proceeds via unidentate intermediates like

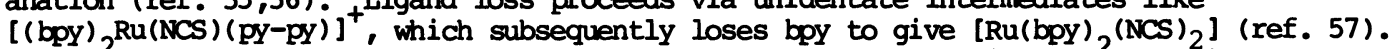
In a later study in dichloromethane both lifetime and photochemical quantum yields for ligand loss were observed for $\left[\mathrm{Ru}(\mathrm{bpy})_{3}\right]^{2+}$ as a function of temperature for a series of anions (ref. 58). From those results the photochemial reaction scheme in Fig. 2 was proposed to account for the observed reactivity. In the absence of direct spectroscopic evidence for the dd state, it is not known whether it or $3_{\mathrm{MLCT}}$ lies lower in energy for $\left[\mathrm{Ru}(\mathrm{bpy})_{3}\right]^{2+}$.

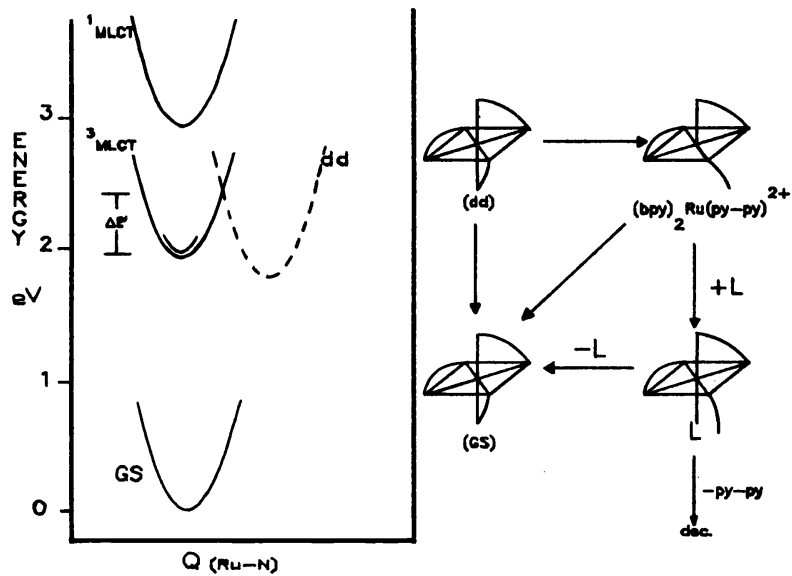

(A)
Fig. 2A. Energy vs. Ru-N displacement for $\left[R u(b p y)_{3}\right]^{2+}$ illustrating the relative dispositions of the three lowest and fourth 3 MLCT states, the lowest $1_{\text {MLCT }}$ state and the energy barrier to the 3 MLCT $\rightarrow$ dd transition. The relative energy of the dd state is not known.

Fig. 2B. A schematic diagram illustrating ring opening and subsequent reactions from the dd state.

Based on this scheme different kinetic limits were proposed to explain the temperature dependence data. In one, the MLCT and dd states are in dynamic eqilibrium as suggested by Van Houten and Watts and $\triangle E^{\prime}$ is the energy difference between $3 \mathrm{MLCT}$ and dd. In the other the $3 \mathrm{MLCT} \rightarrow$ dd transition is an irreversible surface crossing and $\triangle E^{\prime}$ the energy of activation for the process. For completely chelated complexes like [Ru(bpy) ${ }_{3}{ }^{2+}$, even though photosubstitution and chelate opening can be relatively, efficient in solvents, like water, subsequent chelate ring closure, [( bpy $\left.)_{2} \mathrm{Ru}\left(\mathrm{H}_{2} \mathrm{O}\right)(\mathrm{py}-\mathrm{py})\right]^{2+} \rightarrow\left[(\mathrm{bpy})_{2} \mathrm{Ru}(\mathrm{bpy})\right]^{2+}+\mathrm{H}_{2} \mathrm{O}$, provides a useful pathway for "annealing" the system and avoiding ligand loss. 
Although the photochemical mechanism implied in Fig. 2 appears to be appropriate for [Ru(bpy $\left.)_{3}\right]^{2+}$, it may be far from adequate to cover all possible cases. For example, for $\left[\mathrm{Ru}(\mathrm{bpy}){ }_{2}^{3}(\mathrm{py})_{2}\right]^{2+}(\mathrm{py}=$ pyridine $)$ and related complexes a striking difference between the temperature dependences for photoanation and lifetime (ref. 59,60) has led to the suggestion that direct population of the dd state(s) may occur from singlet MLCT states following initial MLCT excitation.

The roles of solvent and ligand variations on the themally activated ${ }^{3} \mathrm{MLCT} \rightarrow$ dd transition have been investigated by temperature dependent lifetime studies (ref. $52,61,62$ ). In the solvent dependence study (ref. 6l) the $3 \mathrm{MLCT} \rightarrow$ dd transition was treated as an intramolecular electron transfer process,

$\underset{3 \text { (MLCT) }}{(d \pi)^{5}\left(\pi^{*}\right)^{1} \longrightarrow(d \pi)^{5}(d a-*)^{1}}$

with $\mathrm{k}^{\circ}$ and $\mathrm{E}_{\mathrm{a}}$ being the pre-exponential term and energy of activation for the ligand to metal electron transfer,

$k=k^{\prime}{ }^{\circ} \exp -\left(\triangle E^{\prime} / R T\right)=A \exp -\left(E_{a} / R T\right)$

Significant variations in $k^{\prime}$ and $\triangle E^{\prime}$ are also observed in the series of complexes [cis$\left.\mathrm{Ru}(\text { bpy })_{2}(L)_{2}\right]^{2+}$ where the n8nchromophoric ligand $L$ was varied (ref. 52). Although there are insufficient data to predict how variations in $\mathrm{L}$ influence the relative energies of MLCT and dd states, some trends are discernible: 1) With appropriate variations in the surrounding ligands, for example, by substituting 4,5-diazafluorene for bpy the energy of the dd state can be selectively perturbed leaving the MLCT states relatively unaffected (ref. 63). 2) When $L$ is a good backbonding ligand like $P R_{3}$ or $\infty$, the MLCT state is destabilized relative to the dd state as evidenced by low energy barriers for the MLCT $\longrightarrow$ dd transition $3+$ In fact, the striking observation has been made that in the series [cis-Ru(bpy) $\left.{ }^{(L)}\right]^{2+}$ the quantum yield for ligand loss increases linearly with the MCT emission energy (fef. 64).

The appearance of relatively low lying dd states in polypyridyl complexes of ruthenium, although of some fundamental interest, is a nuisance in the attempt to design new classes of photosensitizers. The dd states are short lived in solution and of little value in a chemical sense. For [Ru(phen) $\left.]_{3}\right]^{2+}$ in $\mathrm{CH}_{2} \mathrm{Cl}_{2}$ at rocm temperature, 988 of excited state decay occurs through the dd state; 258 for $^{2}\left[\mathrm{Ru}\left(\text { bpy) }{ }_{3}\right]^{2+}\right.$ (ref. 58). In addition, the dd states represent a potentially major source of photolnstability. In the series [cis$\left.\mathrm{Ru}(\mathrm{bpy}){ }_{2}(\mathrm{~L})_{2}\right]^{2+}$ (I-py, N-methylimidazole,....) quantum yields for ligand loss of $>\frac{1}{408}$ have been observed (ref. 59,60). The presence of lowlying dd states virtually rules out the possibility of developing a useful family of MLCT-based photosensitizers based on iron. It seems doubtful that any combination of ligands on iron will give both the necessary visible light absorptivity and sufficient destabilization of the did states to raise them above the MLCT states. For example, MLCT excitation of $\left[\mathrm{Fe}(\mathrm{bpy})_{3}\right]^{2+}$ leads to the rapid population and decay $(\tau=0.6 \mathrm{~ns})$ of a lowlying dd state (ref. 65 ).

There are approaches available for designing "dd-free" MLCT sensitizers. The most straightforward is to turn to os where $10 \mathrm{Dq}$ is higher by 308 and, in general, complexes of Os(II) are photoinert. Even with Os(II), dd states can appear induced by: 1) strong backbonding ligands like $\infty$ which destablize MLCT relative to dd, and 2) by decreasing the symmetry of the complex in such a way as to lift the orthogonality of the $\mathrm{d} \pi$ and do-* orbitals (ref. 66). The net effect is to "mix" the $\mathrm{d} \pi$ and do-* orbitals thus decreasing the energy of the dd states. It has been suggested that the lowered symmetry at $\mathrm{Ru}$ in $[R u(t r p y)]^{2+}\left(\operatorname{trpy}=2,2^{\prime}\left(6,6^{\prime}\right), 2^{\prime \prime}\right.$-terpyridine) arising from the inability of the tridentate trpy ligand to span $180^{\circ}$ leads to a lowlying dd state and a very short lifetime at room temperature (ref. 67).

In the mixed chelates $\left[R u(L-L)_{3-n}(b p y)_{1}\right]^{2+}\left(n=1,2 ;\right.$ I-L is $2,2^{\prime}$-bpyrazine or $2,2^{\prime}$-bisisoquinoline) (ref. $43 \mathrm{~b}, 68$ ), photochemical ligand loss is quenched for the complexes [Ru(LL) (bpy) $]^{2+}$ in part because of the lower lying $\pi *$ levels at $(I-L)$. Studies of this kind show promise for the design of new classes of photochemically stable sensitizers based on Ru.

\section{The fourth MLCT state}

As noted in a previous section, even in the absence of photocemical ligand loss, lifetime studies as a function of temperature reveal the existence of an additional thermally activated process or processes for excited state decay of Os(II) (ref. 41,69) and Ru(II) complexes (ref. $43,68,70$ ).

A contribution to the temperature dependence ocmes from thermal population and decay from a fourth MLCT (ref. 68) which on theoretical grounds is predicted to have a greater amount of singlet character and be more short lived than the three lowest MLCT states (ref. 44). With this interpretation $\triangle E^{\prime}$ is the energy gap to the fourth state and $k$ the rate constant for decay of the fourth state. In contrast to the ${ }^{3} \mathrm{MLCT} \rightarrow$ dd transition where the parameters $\mathrm{k}^{\prime}$ and $\triangle \mathrm{E}^{\prime}$ are characteristically in the range $10^{12}-10^{14} \mathrm{~s}^{-1}$ and 2500-4000 om 1 , for the 
nonphotochemical cases, $\mathrm{k}^{\circ}$ and $\triangle \mathrm{E}^{\prime}$ are generally in the range 107-109 $\mathrm{s}^{-1}$ and 400-1000 $\mathrm{cm}^{-1}$, respectively. As yet, there are insufficient data to establish the factors at the molecular level that dictate the magnitude of the energy splitting between the fourth and lowest three MICT states and the situation is further clouded by the fact that fits of experimental data to Eq. 19 necessarily force the temperature dependence into a single term. In some cases contributions to the experimental data may come from population and decay of both dd and the fourth MLCT states.

\section{Radiative decay of MLCT states}

The availability of an extended series of related complexes based on the same chromophore, e.g., [(bpy)Os(L) $]^{2+}$, offers the possibility of exploring some of the most fundamental processes available to excited states. Experimentally, it is far simpler to carry out such studies based on polypyridyl complexes of Os(II) rather than Ru(II). Although the properties of the MLCT excited states are entirely analogous, it is necessary to carry out temperature dependent studies for $\mathrm{Ru}$ in order to separate out contributions to nonradiative decay from population and decay of dd states.

In a radiative transition energy conservation is achieved by emission of a photon and from Eq. 9 the magnitude of $k_{r}$ is proportional to the square of the transition dipole moment and to $\mathrm{E}^{3}$. Experimentally, radiative $\left(k_{r}\right)$ and nonradiative $\left(k_{n r}\right)$ decay rate constants are detemined by a combination of lifetifhe $(\tau)$ and emission quantum yield $\left(\phi_{e}\right)$ measurements.

$1 / \tau_{0}=k_{r}+k_{n r} ; \phi_{e}=k_{r} \tau_{0}$

The expression for $\phi_{e}$ in Eq. 22 assumes that the efficiency of formation of the emitting state following excitation is unity.

For the majority of MLCT excited states which have been studied $\phi_{\text {is }}$ is $1<108$ at rocm temperature) and difficult to measure accurately which limits the precision of $k_{r}$ Nonetheless, comparisons amongst a series of related complexes like [(bpy)Os(L) ${ }^{r}{ }^{2+}$ show the existence of some systematic trends: 1) Although a considerable scatter appears in the data, $k_{r}$ increases roughly with $\langle\bar{\nu}\rangle^{-1}$ as predicted by Eq. 9 (ref. 46,71 ). The linear increase shows that the transition dipole mament must remain relatively constant through the series. 2) There is no electronic distinction between bpy and phen as chromophoric ligands (ref. 46) which is consistent with the results of molecular orbital calculations which show that the $8 \mathrm{~N}$ character and energies of the lowest lying $\pi$ * orbitals are similar (ref. 48). 3) The transition dipole moment integral can be evaluated from the slope of the plot of $\mathbf{k}_{r}$ vs. $\left\langle v^{3}\right\rangle^{-1}$ and from it an estimate of the molar extinction coefficient_pf the absorption band corresponding to emission can be obtained which for $O s\left(e_{\mathrm{max}} 400 \mathrm{M}^{-1} \mathrm{~cm}^{-1}\right)$ is in reasonable agreement with values estimated from the low temperature single crystal

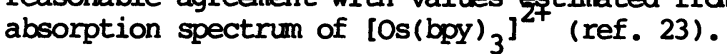

Nonradiative decay In order for a nonradiative decay process to occur with energy conservation, the energy change associated with the change in electronic configuration between the ground and excited states must appear in the surrounding vibrations. The problem in excited state decay is energy disposal and only those vibrations for which $Q$ or $\omega$ are different between the ground and excited states can accept the released energy. The rate constant $k_{r r}$ is the product of two factors (ref. 72-75): 1) A vibrationally induced electronic foupling term. If the Born-Oppenheimer approximation is assumed the to be valid, ground and excited states are orthogonal solutions of the same Hamiltonian and can not mix. In fact, molecular vibrations do lead to slight perturbations in electronic structure. For selected vibrations (the "promoting" modes) one consequence of the perturbation that they exert is a mixing of the excited and ground states which allows the transition between states to occur. 2) vibrational overlap or Franck-condon integrals. The fractional distribution of energy released throughout the "acceptor" modes for which $\triangle \mathrm{Q}$ or $\triangle \omega \neq 0$ depends on the magnitudes of the Franck-Condon overlap integrals (Eq. 14) subject to the constraints imposed by energy conservation.

In the limit of a single promoting mode or of an average contribution from a series of modes, the vibrationally induced electronic coupling term is given by

$B_{0}=c_{k}^{2} \omega_{k}(1 s)(\pi / 2)^{1 / 2} /\left(1000 \mathrm{~cm}^{-1}\right)$ In Eq. $23 \omega_{k}$ is the angular frequency of the promoting mode and the vibronic integral $c_{k}$ is
defined in Eq. 24 (ref. 46 ).

$C_{k}=h\left\langle\Psi_{i}\left|\left(\partial / \partial Q_{k}\right)\right| \Psi_{f}\right\rangle=v_{k}\left(2 / \hbar \omega_{k}\right)^{1 / 2}$

In Eq. $24 \Psi_{i}$ and $\Psi_{f}$ are the products of the electronic wave functions and the vibrational wave function for the promoting mode, $\psi=\psi x, Q_{k}$ is the displacement for the promoting mode, and the integral $v_{k}=\left\langle\psi_{i}|v| \psi_{\mathbf{f}}\right\rangle=\left\langle\psi_{\mathbf{i}}\left|\partial / \partial Q_{k}\right| \psi_{\mathbf{f}}\right\rangle$ contains only non-Born-Oppenheimer
operators. 
The vibrational overlap integrals describe how the energy is initially partitioned amongst the various intramolecular modes when the excited to ground state transition occurs. For the MICT excited states radiative and nonradiative decay occur from the same states so that from the Resonance Raman and emission spectral fitting studies mentioned earlier contributions must exist from 7-8 medium frequency polypyridyl ring stretching vibrations, low frequency ring and metal ligand stretching vibrations, and the solvent. The contributions from medium and low frequency modes can be treated as averages through $S_{M}, \hbar \omega_{M}$, and $S_{L}$, hw obtained by emission spectral fitting. If for the energy gap between the excited and ground states, $E \gg S_{M} \hbar \omega_{M}$, and if $\hbar \omega_{M} \gg k_{B} T(\sim 200 \mathrm{kcal} / \mathrm{mole}$ at $R T)$, it is possible to evaluate the Franck-Condon integrals, Eq. 14 , which gives for $k_{n r}$ (ref. $46,79,81)$.

$$
\begin{aligned}
& k_{n r}=B_{0} \cdot F=B_{0} \cdot\left(1 / h_{\omega_{M}} E_{0}\right)^{1 / 2} \exp \left(-S_{m}\right) \exp \left[-\left(\gamma E_{O} / \hbar \omega_{M}\right)+(\gamma+1)^{2}\left(\Delta \nu_{1 / 2} / s \hbar \omega_{M}\right)^{2} / 16 \ln 2\right] \\
& \gamma=\ln \left(E_{O} / S \hbar \omega_{M}\right)-1
\end{aligned}
$$

Equation 25 includes contributions from: 1) A dominant acceptor mode ( $S_{M}, \hbar \omega_{M}$ ). 2) Low frequency intramolecular and solvent modes through $\Lambda_{\bar{v}}^{\frac{1}{2}}$. In the classical limit $\Delta \bar{v}_{\frac{1}{2}}$ is
given by,

$\Delta \bar{v}_{\frac{1}{2}}=\left[16 \operatorname{kTln} 2\left(x_{0}+x_{i}^{\prime}\right)\right]^{\frac{1}{2}}=\left[2310\left(x_{0}+x_{i}^{\prime}\right)\right]^{\frac{1}{2}}\left(\right.$ at RT in $\left.\mathrm{cm}^{-1}\right)$

In Eq. $27 x_{0}$ is the contribution from solvent and $x_{i}^{\prime}=\sum_{j} x_{i, j}^{\prime}$ is the

contribution from the intramolecular vibrations; the sumation is over all of the contributing low frequency modes with the prime denoting that the higher frequency modes are excluded. 3) An energy gap term $E_{0}$ which is related to the 0-0 energy difference between ground and excited states, $\mathrm{E}_{\infty}$, by,

$E_{0}=E_{\infty}-x_{i}^{\prime}-x_{0}$

$\mathrm{E}_{\mathrm{O}}$ is the energy at which the $\mathrm{v}_{\mathrm{M}}^{*}=0 \rightarrow v_{M}=0$ transition occurs in the emission spectrum for mode $M$ and can be obtained by entission spectral fitting. It is always at higher energy than the apparent emission energy maximum in a structureless emission manifold.

Equations like 25 predict that $l n k$ should vary approximately linearly with the energy gap, and have been referred to as "the enfergy gap law" (ref. 72-75). In the limit $\mathrm{E}_{0}>\mathrm{S}_{\mathrm{M}} \mathrm{h} \omega_{\mathrm{M}}$ energy dissipation through $\omega_{M}$ is dominant and the energy gap term, $\left(E_{O} / h \omega_{M}\right) \sim v_{M}$, dictates in turn the vibrational quantum number of the particular vibrational level which dominates the energy acceptor role. $\underline{k}_{n r}$ increases as $E_{0}$ decreases because vibrational overlap is enhanced for smaller values of $\mathbf{v}$. Although vibrational levels with high $\underline{-}_{1}$ values of $v_{M}$ would be requirfed for a single acceptor mode, e.g., $v_{M}=10$ for $E_{0}=15,000$ an ${ }^{-1}$ and $\hbar \omega_{M}=$ $1500 \mathrm{~cm}^{-1}$, in fact, with 7-8 $v$ (bpy) modes available, the decay process is dominated by transitions in which energy is dissipated into combinations of the $v(b p y)$ modes each of which has a relatively small value of $v$. Contributions from low frequency modes and the solvent are included classically in the $\Delta \bar{v}_{1}$ term. Although they play a role in excited state decay, the fraction of energy dissipated through these modes is much smaller $(<108)$.

The linear relation between $l_{n k}$ and the energy gap predicted by the energy gap law has been observed for families of aromatic hydrocarbons (ref. 73,76,77). However, perhaps the most systematically revealing cases have come from studies of nonradiative decay in a series of MLCT excited states of $\mathrm{Ru}(\mathrm{II}), \mathrm{OS}(\mathrm{II})$, and $\mathrm{Re}(\mathrm{I})$ where linear correlations between Ink and $E_{e m}$ have been observed: 1) for families of related complexes like [(phen)Os(L) ]2+ (ref. 46,78), or [(bpy) $\left.\operatorname{Re}(C))_{3}(L)\right]^{7}$ (ref. 79$)\left(L=p y, P R_{3}, R C N, \ldots\right)$ where the basts for the chromophore is maintained and the energy gap varied by changes in L. 2) for [Os(phen) $]^{2+}$ and a related derivative, by varying the counter ion in dichlromethane

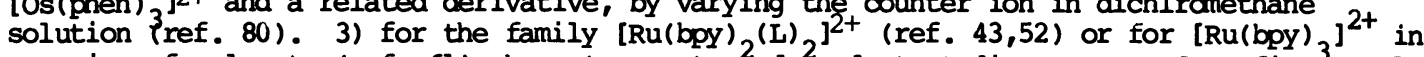
a series of solvents (ref. 61) where temperature dependent studies were used to disentangle contributions to excited state decay from MLCT and dd states. 4) for the families $\left[(\text { bpy }) O s(L)_{4}\right]^{2+}$ or $\left[(\text { phen }) O s(L)_{1}\right]^{2+}$ where the energy gap was changed by solvent variations (ref. 81). 5) for $\left[\mathrm{OS}(\mathrm{bpy}){ }_{2}(\mathrm{CO})(\mathrm{py})\right]^{2+}$ in the glass to fluid transition region in $4: 1(\mathrm{~V} / \mathrm{V})$ EtOH/MeOH $(90-140 \mathrm{~K})$ where the decrease in emission energy with temperature is parallelled by increases in $l_{n k}$ (ref. 82).

In the energy gap law experiments based on MLCT excited state of OS(II) not only are linear

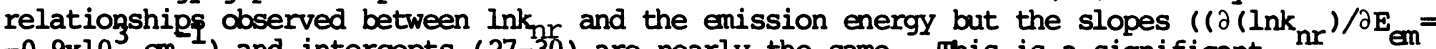
$-0.9 \times 10^{3} \mathrm{~cm}^{-1}$ ) and intercepts (27-30) are nearly the same. This is a significant observation since it suggests that the chromophoric basis for the excited states remains the same throughout the series without significant variations in the electronic coupling term B ' that the same pattern of acceptor vibrations is involved throughout the series, and that the energy gap law holds for MLCT decay regardless of how the energy gap is varied at the microscopic level.

Nonetheless, the observation of a linear relationship between $l n k$ and $E_{\mathrm{nm}}$ or $\mathrm{E}_{0}$ only demonstrates a qualitative agreement with the underlying theory. "Equation 25 is ${ }^{\circ}$ not a 
simple function of $\mathrm{E}_{\mathrm{O}}$ as shown by the appearance of the term containing $\left(\mathrm{E}_{\mathrm{O}}\right)^{-\frac{1}{2}}$ and the dependence of $S_{M}$ on $E_{0}$. Consequently, there is no quantitative information in the slopes and intercepts of simple plots of $\mathrm{lnk}_{\mathrm{nr}}$ vs. $\mathrm{E}_{\mathrm{o}}$.

A far more insightful approach is to use the complete results of emission spectral fitting and the parameters $S_{M}, h \omega_{M}, E_{0}$, and $\Delta \bar{v}_{\frac{1}{2}}$ to calculate the vibrational overlap term $F$ in Eq. 25. Based on such calculations for a series of bpy and phen complexes of os(II) and the relationship, $l_{n k}=\ln \beta_{O}+\operatorname{lnF}$ from Eq. 25, a remarkably detailed insight is gained into the nonradiative decay process (ref. 46): 1) The major contributor (>908) to the vibrational overlap term $F$ comes from the medium frequency $\nu$ (bpy) modes and the term $-\gamma E / \hbar \omega_{M^{*}}$ 2) The slope of a plot of lnk $v s$. InF is unity as predicted by Eq. 25. 3) Both bpy and phen as chromophoric ligands lie on the same plot of $\operatorname{lnk}_{n r}$ vs. InF showing that the electronic coupling term $B$ is the same for both consistent with the results of the molecular orbital calculations alluded to earlier (ref. 44). The increase by a factor of 3 in $k_{p r}$ for bpy compared to phen in analogous complexes, arises because of systematically smaller values of $\mathrm{E}$ and larger values of $\mathrm{S}_{\mathrm{M}}$, 4) From Eq. 25 the intercept of a plot of lnk vs. InF gives ${ }^{\circ} \ln \beta_{O} \sim 34, B_{0} \sim 6 \times 10^{14} \mathrm{~s}^{-1}$ and from Eg. 24, $v_{k} \sim 1300 \mathrm{om}^{-1}$. A value of this magnitude for $v_{k}$ is in line with estimates mades from absorption band intensities for singlet $\rightarrow$ singlet tratisitions which are dipole forbidden but vibronically allowed (ref. 83). The near constancy in B implied by the linear correlation between Ink and F suggests that the pattern of lowlying ${ }^{\mathrm{MLCT}}$ states must remain nearly the same throughout the series of chromophores.

The quantitative success of Eq. 25 is quite striking and its implications profound. As a result of the analysis once $B_{0}$ is evaluated for a particular class of chromophore, $k_{n r}$ can be calculated to within a factor of $\sim 3$ by emission spectral fitting. The situation $\frac{n \pi}{1 s} \frac{\text { much }}{m a}$ Iike that for the appearance of metal to metal charge transfer bands in mixed-valence dimers like [(bpy) $\left.{ }_{2} \mathrm{ClRuII}\left(4,4^{\prime} \text {-bipyridine) Ru III } \mathrm{Cl} \text { (bpy }\right)_{2}\right]^{3+}$ where absorption band spectral profiles have been used to estimate rate constants for intramolecular electron transfer (ref. 10a,8485). In either case the spectral profiles for the appropriate spectroscopic process, $($ bojy) $\mathrm{Ru} I I I \rightarrow$ (bpy) $\mathrm{Ru}$ II or $\mathrm{Ru}$ II $\mathrm{Ru}$ III $\rightarrow \mathrm{Ru}$ III-RuII, contain all the needed information required to calculate the vibrational contributions to the electron transfer or nonradiative decay rate constants.

Medium effects From Eq. 25 the solvent plays a minor role as energy acceptor in the initial non-radiative decay process. Following the transition between states and subsequent vibrational relaxation, the energy does ultimately end up in the surrounding solvent thermal pool.

The solvent also plays a role in determining the magnitude of $k_{n r}$ through the energy gap term $\gamma \mathrm{E} / \mathrm{h} \omega_{M}$ which dictates the pattern of $\nu$ (bpy or phen) levels which dominate as energy acceptols. Mor [Ru(bpy) $]^{2+}$ or [Os(bpy) $]_{3}^{2+}$ where a dipole is created in the excited state, the excited state is preferentially stabllized relative to the ground state by the surrounding solvent dipoles as shown by the fact that $\mathrm{E}$ tends to decrease as the static dielectric constant of the solvent increases (ref. 86). ${ }^{\circ}$ As noted above, the changes in $E_{0}$ are tracked by lnk as predicted by the energy gap law. However, the microscopic origins of the solvent shifts observed in $\mathrm{E}_{0}$ are not understood (ref. 81,86) and dielectric continuum theory, which does provide a basis for accounting for solvent shifts for MLCT absorption bands (ref. 39 ), is not successful for $\mathrm{E}_{\mathrm{em}}$ (ref. 86).

Experimental evidence is available to suggest that in hydroxylic solvents, especially $\mathrm{H}_{2} \mathrm{O}$ or $\mathrm{CH}_{3} \mathrm{OH}$, high frequency $v(\mathrm{O}-\mathrm{H})$ modes at $\sim 3500 \mathrm{~cm}^{-1}$ can also play a role as energy acceptors in nohradiative decay ( $r e f, 81,86$ ). The evidence includes the fact that for the family of complexes [Os(phen)(L) j2t in both polar organic and hydroxylic solvents, plots of lnk nr $_{\text {vs. }}$ Eem show that excited state decay is always faster in the hydroxylic solvents and that a significant $\mathrm{k}_{2} \mathrm{O} / \mathrm{k}_{\mathrm{D}_{2} \mathrm{O}}$ kinetic isotope effect exists $(\sim 2)$. Even though $\mathrm{S}$ is small for such vibrations, they make a contribution because of their relatively high frequencies $(\sim 3500$ $\mathrm{cm}^{-1}$ ) (ref. 86).

The emission and lifetime characteristics of MLCT excited states, most notably for $\left[\mathrm{Ru}(\text { bpy })_{3}\right]^{2+}$, have been investigated in a variety of media ranging from crystals to glasses and polymeric films (ref. $34,41,70,87-90$ ). In such media where dipole reorientations in the surrounding medium are restricted and different chemical environments may exist for the excited state, non-exponential decay kinetics are often observed and intramolecular processes such as the MLCT $\rightarrow$ dd transition can be inhibited. An important contributor to such effects is, no doubt, the fact that in a hard glass, polymeric film, or the solid state, dipole reorientation times are long on the time scale for excited state decay. Dipole orientations surrounding the molecule are necessarily constrained to be those appropriate to the electronic configuration of the ground state. By contrast, in solution solvent dipole reorientation times are shorter than excited state lifetimes for all excpt the shortest lived excited states. The solvent dipoles have the opportunity to reorient to configurations appropriate to the electronic configuration of the excited state before it decays. Consequently the excited state is stabilized in fluid solution compared to a rigid medium. 
In glasses, in the intermediate temperature range between the hard glass and fluid states, interesting time dependent phencmena can be observed as the correlation times for dipole reorientations approach the time scale for excited state decay (ref. 91-92). In this domain a complex time dependence for excited state decay is predicted since as solvent dipoles reorient a time dependence is introduced into the energy gap, $E_{0}=E_{0}(t)$ leading to a time dependence in $k_{n r}, l n k_{n r}(t) \propto-\gamma E_{0}(t) / h \omega$, Eq. 25. In this limit measurements taken at a single wave length give rise to nonexponential decay kinetics but dipole reorientation and excited state decay can be deconvoluted using the full emission spectrum as a function of time. Based on the deconvolution, excited state decay measurements can be used to probe the dynamics of dipole reorientation as the glass softens.

\section{SYNTHETIC DESIGN OF EXCITED STATE PROPERTIES}

Fram the synthetic point of view, the availability of MLCT chromophores based on variations in the chromophoric and non-chromophoric ligands (ref. 43,60,93-96) is quite remarkable since it offers the possibility of "designer" excited states where photophysical and photochemical properties can be controlled in a systematic way by synthetic variations. As a result of the photochemical and photophysical studies described here and the remarkably consistent patterns that emerge, it is possible to formulate a set of "rules" that can both account for excited state properties and guide the design of new complexes. 1) Radiative decay rates vary approximately with $\mathrm{Eem}^{3}$. For complexes of Os(II) and Ru(II) having the same emission energies, $k_{r}$ for $O s$ will be greater by $\sim 3$ because of the higher spin-orbit coupling constant for Os. ${ }^{2}$ ) Nonradiative decay is dominated by the energy accepting role of a series of polypyridine-based ring stretching vibrations. For equivalent bpy and phen complexes the bpy complexes undergo more rapid non-radiative decay by a factor of $\sim 3$ because of smaller values of $E_{\mathrm{O}}\left(\sim \mathrm{E}_{\mathrm{em}}\right)$ and larger values of $\mathrm{S}_{\mathrm{M}}$. Non-radiative decay rates are more rapid in $\mathrm{H}_{2} \mathrm{O}$ than in polar organic solvents because of contributions fram $v(\mathrm{O}-\mathrm{H})$ modes as energy acceptors. 3) The emission energy is the deciding factor in determining excited state properties (ref. 14a). In a family of related complexes where $\langle d\rangle$ and $B$ remain nearly constant, $k_{r}$ varies with $E_{e m}, k_{r}$ with $\exp \left(-E_{e m}\right)$ and $\phi_{e}$ with $k_{r}$ and $k_{\text {nr }}$. 4) Excited state redox potentfals can also be estiffited from the emission energy using ground state redox potentials as shown in Eq. 29.

$$
\begin{aligned}
& E^{O^{\prime}}\left[(\text { phen }) O s(L){ }_{4}^{2+* /+}\right]=E^{O^{\prime}}\left[(\text { phen }) O s(L)_{4}^{2+/ t}\right]+E_{E m} \\
& E^{O^{\prime}}\left[(\text { phen }) O s(L)_{4}^{3+/ 2+*}\right]=E^{O^{\prime}}\left[(\text { phen }) O s(L)_{4}\right)^{3+/ 2+)]-E_{e m}}
\end{aligned}
$$

5) Since the ligand-based [(phen) $\left.Q s(L)_{4}\right]^{2+/+}$ couple is relatively insensitive to variations in $\mathrm{L}, \mathrm{E}_{\mathrm{em}}$ varies linearly with $\mathrm{E}^{\mathrm{O}}$ for ${ }^{4}$ the $\mathrm{M}(\mathrm{III} / \mathrm{II})$-based couple. As a consequence, in $\mathrm{a}$ closely related series of complexes, excited state emission energies, lifetimes, and emission effencies can all be estimated simply by measurement of the potential of the ground state OS(III/II) couple (ref. 14a). 6) In complexes of Ru(II) complications exist from lowlying dd states and unravelling their contributions to excited state properties demands temperature dependent lifetime studies. As yet, there are insufficient data and an absence of the theoretical insight needed to make clear predictions about how relative energies of the MLCT and dd states vary with variations in ligands. However, in certain mixed chelates containing at least one good polypyridyl-based $\pi$-accepting ligand, the energy gap between the MLCT and IF states can be sufficiently large that the population of dd states is relatively insignificant at room temperature. Photochemical ligand loss is inhibited for chelates where the chelate can reclose following dissociation and is favored in polar organic solvents in the presence of cordinating anions like $\mathrm{Cl}^{-}$which can capture open coordination sites.

Acknowledgement I would like to acknowledge support of my own work in this area by the U.S. Department of Energy and of the contributions of J. V. Caspar and E. M. Kober whose work has provided much of the basis for this article.

\section{REFERENCES}

1. T. P. Paris and W. W. Brandt, J. Am. Chem Soc., 81, 5001 (1959).

2. a) H. D. Gafney and A. W. Adamson, J. Am. Chem Soc., 94,8238 (1972); b) T. N. Demas and A. W. Adamson, J. Am. Chem. Soc., 95, 5159 (1973).

3. a) C. R. Bock, T. J. Meyer and D. G. Whitten, J. Am. Chem. Soc., 96, 4710 (1974); b) R. C. Young, T. J. Meyer and D. G. Whitten, J. Am. Chem. Soc., 97, 781 (1975).

4. G. Navon and N. Sutin, Inorg. Chem., 13, 2159 (1974).

5. a) C. P. Anderson, D. J. Salmon, T. J. Meyer and R. C. Young, J. Am. Chem. Soc. 99, 1980 (1977); b) M. Maestri and M. Grätzel, Ber. Bunsenges. Phys. Chem., 81, 504 (1977).

6. C. R. Bock, T. J. Meyer and D. G. Whitten, J. Am. Chem. Soc., 97, 2909 (1975).

7. a) C. R. Bock, J. A. Connor, A. R. Gutierrez, T. J. Meyer, D. G. Whitten, B. P. Sullivan and J. K. Nagle, J. Am. Chem. Soc., 101, 4815 (1979); Chem. Phys. Lett., 61, 522 (1979); b) R. Ballardini, G. Varani, M. T. Indelli, F. Scandola and v. Baizani, J. Am. Chem. Soc., 100, 7219 (1978).

8. a) N. Sutin and C. Creutz, Pure Appl. Chem., 52, 2717 (1980); b) N. Sutin, J. Photochem., 10, 19 (1979). 
9. a) V. Balzani, F. Bolleta, M. T. Gandolfi and M. Maestri, Top. Curr. Chem., 75, 1 (1978); b) K. Kalyanasundaram, Coord. Chem. Rev., 46, 159 (1982).

10. a) T. J. Meyer, ACc. Chem. Res., 11, 94 (1978); T. J. Meyer, Prog. Inorg. Chem., 30,389 (1983); C) D. G. Whitten, Acct. Chem. Res., 13, 83 (1980).

11. D. Rehm and A. Weller, Ber. Bunsenges. Phys. Chem., 13,834 (1969); Isr. J. Chem。, $8,259(1970)$.

12. a) G. A. Crosby, Acc. Chem. Res. , 8, 231 (1975); b) M. K. DeAmond and C. M. Carlin, Coord. Chem. Rev, 36,325 (1981); c) T. J. Kemp, Prog. Read. Kinetics, 10, 301 $(1980)$; d) J. Ferguson, F. Ferren, E. R. Krauss, M. Maeder and J. Vrbancich, Coord. Chem. Rev., 64, 21 (1985).

13. a) T. Saji and S. Aoyaqui, J. Electroanal. Chem., 60, 1 (1975); 58, 401 (1975); b) V. Sharda, M. J. Cook, A. P. Ievis, G. S. G. McAuliffe, A. J. Thomson, and D. J. Robbins, J. C. S. Perkin Trans. II, 1309 (1984).

14. a) E. M. Kober, J. L. Marshall, W. J. Dressick, B. P. Sullivan, J. V. Caspar and T. J. Meyer, Inorg. Chem., 24, 2755 (1985); b) E. S. Dodsworth and A. B. P. Lever, Chem. Phys. Lett., 119,61 (1985).

15. R. B. Wilson and E. I. Solamon, J. Am. Chem. Soc., 102, 4085 (1980)

16. D. Guenzburger, A. Garnier and J. Danon, Inorg. Chim. Acta.. 21, 119 (1977).

17. C. J. Ballhausen, Introduction to Ligand Fields, MoGraw-Hill, New York (1962).

18. A. B. P. Lever, Inorganic Electronic Spectroscopy (2nd ed.), Elsevier, New York (1984).

19. a) P. Belser, A. Von Zelewsky, A. Juris, F. Barigeletti, F. Tucci and V. Balzani, Chem. Phys. Lett., 89, 101 (1982); b) F. Barigelletti, P. Belser, A. von Zelewsky and v. Balzani, J. Phys. Chem. 89, 3680 (1985).

20. a) T. Ohno and S. Kato, Bull. Chem. Soc. Jpn., 57, 3391 (1984); b) V. T. Cocmbe, G. A. Heath, A. J. Mackenzie and L. J. Yellowlees, Inorg. Chem., 23, 3423 (1984).

21. M. Nishizawa, T. M. Suzuki, S. Sprouse, R. J. Watts and P. C. Ford, Inorg. Chem. 23, 1037 (1984).

22. a) G. M. Bryant, J. E. Fergusson and J. K. J. Powell, Aust. J. Chem., 24, 257 (1971); G. M. Bryant and J. E. Fergusson, Aust. J. Chem., 24, 275 (19971)'.

23. a) F. Felix, J. Ferguson, H. U. Gudel and A. Ludi, Chem. phys. Lett., 62, 153 (1979); b) F. Felix, J. Ferguson, H. U. Gudel and A. Ludi, J. Am. Chem. Soc., 102, 4096 (1980); S. Decurtins, F. Felix, J. Ferguson, H. U. Gudel and A. Ludi, J. Am. Chem. Soc., 102,4102 (1980).

24. E. M. Kober and T. J. Meyer, Inorg. Chem., 21, 3967 (1982).

25. J. Ferguson and F. Herren, Chem. Phys., 76,45 (1983).

26. A. D. Kirk, P. E. Hoggard, G. B. Porter, M. G. Rockley and M. W. Windsor, Chem. Phys. Lett.. 37, 199 (1976).

27. J. N. Demas and D. G. Taylor, Inorg. Chem., 18, 3177 (1979).

28. P. G. Bradley, N. Kress, B. A. Hornberger, R. F. Dallinger and W. H. Woodruff, J. Am. Chem. Soc., 103, 7441 (1981).

29. a) M. Forster and R. E. Hester, Chem. Phys. Lett., 81,42 (1981); b) W. K. Smothers and M. S. Wrighton, J. Am. Chem. Soc., 105, 1067 (1983).

30. C. M. Carlin and M. K. DeAmond, Chem. Phys. Lett., 89, 297 (1982); J. Am. Chem. Soc., 107, 53 (1985).

31. J. V. Caspar, T. D. Westmoreland, G. H. Allen, P. G. Bradley, T. J. Meyer and W. H. Woodruff, J. Am. Chem. Soc., 106, 3492 (1984).

32. G. A. Heath, L. J. Yellowlees and P. S. Braterman, J. C. S. Chem. Comm., 287 (1981); Chem. Phys. Lett., 92, 66 (1982).

33. D. E. Morris, K. W. Hanck' and M. K. DeAmond, J. Am. Chem. Soc., 103, 3032 (1983).

34. a) H. Yersin and E. Gallhuber, J. Am. Chem. SoC., 106, 6582 (1984); b) J. Ferguson and E. R. Krausz, Chem. Phys. Lett., 93, 21 (1983).

35. a) R. F. Dallinger, Inorg. Chem, in press; b) S. J. Milder, J. S. Gold and D. S. Kliger, J. Phys. Chem., 90, 548 (1986)

36. a) C. Creutz, Prog. Inorg. Chem., 30, i (1983); b) K. Y. Wong and P. N. Schatz, Prog. Inorg. Chem, 28369 (1981); c) N. S. Hush, Prog. Inorg. Chem., 8,391 (1967); Electrochim. Acta, 13,1005 (1968).

37. P. S. Braterman, G. A. Heath, L. J. Yellowless, J. C. S. Dalton, 1081 (1985).

38. R. A. Marcus, J. Chem. Phys., 43, 1261 (1965); J. Chem. Phys., 39, 1734 (1963).

39. E. M. Kober, B. P. Sullivan and T. J. Meyer, Inorg. Chem., 23, 2098 (1984).

40. a) D. E. Lacky, B. J. Pankuch and G. A. Crosby, J. Phys. Chem, , 84, 2068 (1980); G. D. Hager and G. A. Crosby, J. Am. Chem. Soc., 97, 7031 (1975); G. D. Hager, R. J. Watts and G. A. Crosby, J. Am. Chem. Soc., 97, $7037^{\prime}$ (1975).

41. S. R. Allsopp, A. Cox, T. J. Kemp, W. J. Reed, V. Carassiti and O. Traverso, J. Chem. Soc. Faraday Trans. 1, 75, 353 (1979).

42. a) R. S. Lumpkin and T. J. Meyer, J. Phys. Chem., in press; b) E. G. Megehee and T. J. Meyer, in preparation.

43. a) G. H. Allen, R. P. White, D. P. Rillema and T. J. Meyer, J. Am. Chem. Soc. 106,2613 (1984); b) F. Barigeletti, F. Juris, V. Balzani, P. Belser and A. vón zelewsky, Inorg. Chem., 22, 3335 (1983).

44. E. M. Kober and T. J. Meyer, Inorg. Chem., 23, 3877 (1984).

45. E. M. Rober and T. J. Meyer, in preparation.

46. E. M. Kober, J. V. Caspar, R. S. Lumpkin and T. J. Meyer, J. Phys. Chem,, in press.

47. a) M. H. Chisholm, J. C. Huffman, I. P. Rothwell, P. G. Bradley, N. Kress and W. H. Woodruff, J. Am. Chem. Soc., 103, 4945 (1981); b) L. J. Radonovich, M. W. Eyring, T. J. Groshens and K. J. Klabunde, J. Am. Chem. Soc., 104, 2816 (1982).

48. E. M. Kober and T. J. Meyer, Inorg. Chem., 24, 106 (1985).

49. O. Poizet and C. Sourisseau, J. Phys. Chem., 88, 3007 (1984).

50. H. Yersin, H. Otto, J. I. Zink and G. Gliemann, J. Am. Chem. Soc., 102, 951 (1980); G. Herzberg, Molecular Spectra and Molecular Structures. I. Spectra of Diatomic Molecules (2nd Ed.), pp. 20 and 200, Van Nostrand Reinhold, New York (1950).

51. C. J. Ballhausen, Molecular Electronic Structures of Transition Metal Complexes, Chap. 4, MoGraw-Hill, New York (1979). 
52. J. V. Caspar and T. J. Meyer, Inorg. Chem., 22, 2444 (1983).

53. a) J. V. Caspar, Ph.D. Dissertation, University of North Carolina, 1983; b) J. V. Caspar, R. S. Lumpkin, E. M. Rober and T. J. Meyer, in preparation.

54. J. Van Houten and R. J. Watts, J. Am. Chem. Soc., 17, 3381 (1978); Inorg. Chem., 17,3381 (1978).

55. M. Gleria, F. Minto, G. Baggiato and P. Bortolus, J. Chem. Soc. Chem. Commun., 285 (1978).

56. a) P. E. Hoggard and G. B. Porter, J. Am. Chem. Soc. 100, 1457 (1978); b) W. M. Wallace and P. E. Hoggard, Inorg. Chem., 19,2141 (19)

57. B. Durham, J. L. Walsh, C. L. Carter and T. J. Meyer, Inorg. Chem., 19, 860 (1980).

58. B. Durham, J. V. Caspar, J. K. Nagle and T. J. Meyer, J. Am. Chem. Soc., 104, 4803 (1982).

59. D. V. Pennick and B. Durham, Inorg. Chem., 23, 3841 (1984).

60. W. M. Wacholtz, R. A. Auerbach, R. H. Schmehl, M. Ollino and W. R. Cherry, Inorg. Chem., 24, 1758 (1985).

61. J. V. Caspar and T. J. Meyer, J. Am. Chem. Soc., 105, 5583 (1983).

62. W. F. Wacholtz, R. A. Auerbach and R. H. Schmehl, Inorg. Chem., 25, 227 (1986).

63. L. J. Henderson, Jr. and W. R. Cherry, J. Photochem., 28,143 (1985).

64 . P. V. Pinnick and B. Durham, Inorg. Chem., 23, 1440 (1984).

65. C. Creutz, M. Chou, T. L. Netzel, M. Okamura and N. Sutin, J. Am. Chem. Soc., 102, 1309 (1980).

66. G. Allen, B. P. Sullivan and T. J. Meyer, J. C. S. Chem. Comm., 793 (1981).

67. J. R. Kirchoff, D. R. MoMillan, P. A. Marnot and J. P. Sauvage, J. Am. Chem. Soc. 107,1138 (1985).

68. G. H. Allen, R. P. White, D. P. Rillema and T. J. Meyer, J. Am. Chem. Soc., 106, 2613 (1984).

69. a) R. S. Lumpkin and T. J. Meyer, in preparation; b) E. Megehee and T. J. Meyer, in preparation.

70. S. R. Allsop, A. Cox, T. J. Remp and W. J. Reed, J. C. S. Faraday I, 74, 1275 (1978).

71. M. J. Cook, A. P. Lewis, G. S. G. McAuliffe, V. Sharda, A. J. Thomson, D. J. Robbins, J. C. S. Perkin Trans. II, 1303 (1984).

72. a) B. R. Henry and W. Siebrand, Organic Molecular Photophysics (Vol. 1), Chap. 4, J. B. Birks, London (1973); F. K. Fong, "Radiationless Processes", Top. Appl. Phys. (Vol. 15), Springer-Verlag, New York (1976); P. Avouris, W. M. Gelbart and M. A. El-Sayed, Chem. Rev., 77, 793 (1977); K. F. Freed, Acc. Chem. Res., 11, 74 (1978); E. C. Lim, Excited States, Academic Press, New York (1979); S. H. Lin, Radiationless Transitions, Academic Press, New York (1980); E. J. Heller and R. C. Brown, J. Chem. Phys., 79, 3336 (1983).

73. G. W. Robinson and R. P. Frosch, J. Chem. Phys., 37, 1962 (1962); G. W. Robinson, J. Chem. Phys., 38, 1187 (1963); G. W. Robinson and C. A. Langhoff, Chem. Phys., 5, 1 (1974).

74. Hunt, G. R., E. F. MoCoy and I. G. Ross, Aust. J. Chem., 15, 591 (1962); J. P. Byrne, E. F. Mocoy and I. G. Ross, Aust. J. Chem., 18, $15 \overline{89}$ (1965).

75. a) W. Siebrand and D. F. Williams, J. Chem. Phys., $\overline{46}, 403$ (1967); b) W. Siebrand and D. F. Williams, J. Chem. Phys., 49, 1860 (1968); c) S. H. Lin, J. Chem. Phys., 44, 3759 (1966); d) S. H. Lin and R. Bersohn, J. Chem. Phys., 48, 2732 (1968).

76. G. D. Gillipsie and E. C. Lim, Chem. Phys. Lett., 63, 193 (1979).

77. a) H. J. Griesser and U. P. Wild, Chem. Phys., 52, 117 (1980); b) A. Maciejewski, A. Safarzadeh-Amiri, R. E. Verrall and R. P. Steer, Chem. Phys., 87, 295 (1984).

78. J. V. Caspar, E. M. Kober, B. P. Sullivan and T. J. Meyer, J. Am. Chem. Soc., 104, 630 (1982).

79. J. V. Caspar and T. J. Meyer, J. Phys. Chem., 87, 952 (1983).

80. W. J. vining, J. V. Caspar and T. J. Meyer, J. Phys. Chem., 89, 1095 (1985).

81. J. V. Caspar, B. P. Sullivan, E. M. Kober and T. J. Meyer, chem. Phys. Lett., 91 , 91 (1982).

82. R. S. Lumpkin and T. J. Meyer, J. Phys. Chem., in press.

83. A. J. Albrecht, J. Chem. Phys. 38, 354 (1963); R. M. Hochstrasser and C. Marzzacco, J. Chem. Phys., 49, $9 \overline{71}$ (1968); G. J. Small and S. Kusserow, J. Chem. Phys., 60,1558 (1974).

84. G. A. Neyhart, K. Goldsby and T. J. Meyer, submitted.

85. a) T. J. Meyer, Chem. Phys. Lett., 64, 417 (1979); b) T. J. Meyer, Inorganic Chemistry: Toward the 2list Century, ACS Symposium Series no. 211, Ed., M. H. Chisholm, Washington, D.C. (1983).

86. J. V. Caspar, E. M. Kober, R. S. Lumpkin and T. J. Meyer, in preparation.

87. D. A. Buttry and F. C. Anson, J. Am. Chem. Soc., 104, 4824 (1982).

88. R. A. Della Guardia and J. K. Thomas, J. Phys. Chem., 87, 990 (1983).

89. J. Wheeler and J. K. Thomas, J. Phys. Chem., 86, 4540 (1982).

90. D. Krenske, S. Abdo, H. Van Damme, M. Cruz and J. J. Fripiat, J. Phys. Chem., 84, 2447 (1980).

91. J. Ferguson, E. R. Krauss and M. Maeder, J. Phys. Chem., 89, 1852 (1985).

92. a) E. Danielson, R. S. Lumpkin and T. J. Meyer, J. Phys. Chem., in press; b) N. Kitamura, H.-B, Kim, Y. Kawanishi, R. Obata and S. Tazuke, J. Phys. Chem., 90, 1488 (1986).

93. V. Balzani, A. Juris, F. Barigelletti, P. Belser and A. von Zelewsky, Proc. Riken Int'l Sym. on Photochem. of Metal Complexes, Sci. Papers Inst. of Phys. and Chem. Res., 78, 78 (1984) and references therein.

94. M. J. Cook, A. P. Lewis and A. J. Thamson, J. C. S. Perkin Trans. II, 1293 (1984).

95. A. Juris, V. Balzani, P. Belser, and A. von Zelewsky, Helv. Chim. Acta, 64, 2175 (1981).

96. A. Baser, M. Weiner, T. C. Strekas and H. D. Gafney, Inorg. Chem., 21, 1085 (1982). 